\title{
Preparation and in vivo evaluation of a gel-based nasal delivery system for risperidone
}

\author{
FUGEN GU ${ }^{1+}$ \\ WEINA MA ${ }^{2}$ \\ GENDALAI MENG ${ }^{1}$ \\ CHUNZHI WU ${ }^{1}$ \\ YI WANG ${ }^{1}$ \\ ${ }^{1}$ Department of Pharmaceutics \\ Affiliated Hospital, Inner Mongolia \\ Medical University, Hohhot 010050 \\ China \\ 2 School of Pharmacy, Inner Mongolia \\ Medical University, Hohhot 010110 \\ China
}

Accepted August 3, 2016

Published online September 6, 2016

\begin{abstract}
The aim of this study was to prepare a nasal gel of risperidone and to investigate the pharmacokinetics and relative bioavailability of the drug in rats. Compared with oral dosing, the risperidone nasal gel exhibited very fast absorption and high bioavailability. Maximal plasma concentration $\left(c_{\max }\right)$ and the time to reach $c_{\max }\left(t_{\max }\right)$ were $15.2 \mu \mathrm{g} \mathrm{mL} \mathrm{m}^{-1}$ and $5 \mathrm{~min}$ for the nasal gel, $3.6 \mu \mathrm{g} \mathrm{mL} \mathrm{m}^{-1}$ and $30 \mathrm{~min}$ for the oral drug suspension, respectively. Pharmacokinetic parameters such as $t_{\max }, c_{\max }$ and AUC of oral and nasal routes were significantly different $(p<0.01)$. Relative bioavailability of the drug nasal preparation to the oral suspension was up to $1600.0 \%$. Further, the in vitro effect of the risperidone nasal gel on nasal mucociliary movement was also investigated using a toad palate model. The risperidone nasal formulation showed mild ciliotoxicity, but the adverse effect was temporary and reversible.
\end{abstract}

Keywords: risperidone, nasal gel, formulation, pharmacokinetics, bioavailability, ciliotoxicity

Risperidone is an atypical second-generation antipsychotic drug. It can act as an antagonist to both D2 and 5-HT2 receptors in the brain and is therefore widely used in the clinical treatment of positive and negative symptoms in patients with schizophrenia. As the compound is water-insoluble and exists in various polymorphic forms, these undesirable physiochemical properties of the drug did not only cause some difficulties in pharmaceutical formulations, but also led to poor and variable oral bioavailability (1). Today, marketed pharmaceutical products of risperidone are mainly oral formulations such as tablets and capsules. Following oral administration, the above preparations often undergo extensive first-pass metabolism, resulting in a slow onset of action and relatively low bioavailability (2). Furthermore, it is very difficult for orally administered risperidone to transport and enter the central nervous system (CNS) because of the existence of the blood brain barrier (BBB); this often caused poor treatment efficacy of the drug. Therefore, oral dosing of risperidone may not be an ideal route in the clinical treatment of schizophrenia.

\footnotetext{
*Correspondence; e-mail: fgczh@sina.com
} 
Only a few decades ago, the potential of intranasal administration to reach the CNS gained great interest and some pharmacological agents following nasal dosing could bypass the BBB and enter the CNS. The advantages of nose-to-brain transport include the avoidance of systemic circulation, reducing the risk of systemic side effects and hepatic/renal clearing, and the possibility of chronic administration. Furthermore, its non-invasiveness, self-administration and the rapid onset of action represent an attractive option to further explore this route of administration to ultimately improve treatment efficacy of many CNS diseases (3). Until now, there have been many drug delivery systems intended for nasal application, such as drops, sprays, powders, gels, emulsions, ointments, etc. Among them, drops and sprays are not ideal dosage forms because of their shorter residence time after nasal dosing and therefore relatively poor absorption and bioavailability. Nasal gels, however, can increase the residence time within the nasal cavity and intensify the contact between nasal mucosa and the drug, which facilitates drug absorption and improves bioavailability. They are thus one of the most ideal delivery systems for nasal administration (4).

So far, the brain targeting behavior of risperidone nanoemulsion following intranasal administration has been confirmed by gamma scintigraphy imaging of rat brain (5). However, the new drug delivery system of risperidone has many limitations, such as complicated prescribing, poor physicochemical stability, and especially shorter nasal residence time. Furthermore, the exact bioavailability of the drug and its nasal ciliotoxicity following nasal dosing were not investigated in the literature. Thus, the objective of the present study was to prepare a risperidone nasal gel and to further investigate the pharmacokinetics and bioavailability of the drug in rats. Also, the in vitro effect of the risperidone nasal preparation on nasal mucociliary movement is explored in this paper as well.

\section{EXPERIMENTAL}

\section{Materials}

Risperidone of $99.9 \%$ purity was purchased from Beijing Gaobo Pharmaceutical Chemical Technology Development Co. (China). Carbopol 940 was purchased from Noveon Inc. (USA). DM $\beta$ CD of $98.3 \%$ purity was a gift from Binzhou Zhiyuan Biotechnology Co., Ltd. (China). Chlorobutanol of $98.0 \%$ purity was purchased from Sinopharm Chemical Reagent Co. Ltd. (China). The HPLC grade acetonitrile was obtained from Fisher Scientific (USA). All other chemicals and solvents were of analytical reagent grade and were obtained from commercial sources and used as received without further purification. Deionized double-distilled water was used throughout.

\section{Preparation of risperidone nasal gel}

Optimized prescription of risperidone nasal gel was obtained on the basis of the results of our previous study and the relevant literature on nasal delivery of medications (6, 7). The drug formulation was prepared using the cold dissolving method (8). Briefly, an exact amount of Carbopol $940(0.35 \%, \mathrm{~m} / \mathrm{V})$ was slowly added to approximately $40 \mathrm{~mL}$ of purified water under continuous stirring, and the resultant dispersion was then kept at 4 ${ }^{\circ} \mathrm{C}$ overnight for complete swelling, after which it was warmed to room temperature, followed by adjustment to $\mathrm{pH} 6.0$ using triethanolamine to obtain a transparent homogenous 
blank gel. Accurately weighed risperidone $(0.5 \%, \mathrm{~m} / \mathrm{V})$ together with chlorobutanol $(0.5 \%$ $\mathrm{m} / \mathrm{V}$ ) was dissolved successively in about $20 \mathrm{~mL}$ of hot propylene glycol, while the required amount of $\mathrm{DM} \beta \mathrm{CD}(15 \%, \mathrm{~m} / \mathrm{V})$ was dissolved in an appropriate volume of purified water. The two solutions were then mixed to obtain a homogeneous solution and the resultant solution was slowly added to the above described blank gel under continuous stirring. Finally, the remaining water was added dropwise into the gel to reach the specified volume of the drug formulation.

\section{Animal experiments}

The study was approved by the Institutional Animal Ethics Committee of Affiliated Hospital, Inner Mongolia Medical University. All procedures and the care of rats were in accordance with the institutional guidelines for animal use in research. Throughout the experiment, the animals were housed in plastic cages on corn-cob bedding in a temperature controlled room $\left(23 \pm 2{ }^{\circ} \mathrm{C}\right)$ with a $12 \mathrm{~h}$ light/dark cycle. The animals were kept in these facilities for at least 1 week before the experiment and fasted for $12 \mathrm{~h}$ prior to experiments with free access to water. Male Wistar rats (Inner Mongolia University Experimental Animal Center, Hohhot, China) weighing 250-300 g were used in the study. Twelve rats were randomly divided into two groups based on the time of blood sampling, each group having six animals. Nasal administration studies were performed according to the method described by Gu et al. (9). The test group rats were first anesthetized with urethane given intraperitoneally and then placed on their backs, after which the trachea was cannulated with a polyethylene tube to stop nasal respiration, and the esophagus was tied to the tube to prevent peroral absorption. Risperidone nasal gel was administered at a dose of $3.0 \mathrm{mg}$ $\mathrm{kg}^{-1}$ b.m. through a unilateral naris. Blood samples of $0.5 \mathrm{~mL}$ each were withdrawn into 1.5-mL heparinized PE tubes from the retro-orbital plexus at 1, 5, 15, 30, 60, 90, 120, 150,180, 240 and $360 \mathrm{~min}$ immediately after dosing of the nasal gel formulation. For the animals of the oral dosing group, risperidone suspension prepared with $0.5 \%$ CMC solution was given at a dose of $30 \mathrm{mg} \mathrm{kg}$-1 b.m. via gastric gavage. Blood samples were also collected at 10, 15, 20, 30, 45, 60, 90, 120, 180, 240 and 360 min following oral dosing. For either nasal or oral route, an equal volume of $0.9 \%$ normal saline was given intraperitoneally to rats immediately after each blood sampling. All plasma samples were obtained by centrifuging blood samples at $13,000 \mathrm{rpm}$ for $10 \mathrm{~min}$ and were stored at $-20^{\circ} \mathrm{C}$ until analysis.

\section{Risperidone analysis in plasma}

Risperidone in rat plasma was quantified by the HPLC-UV method as reported (2), with minor modifications. Briefly, $20 \mu \mathrm{L}$ of $75 \mu \mathrm{g} \mathrm{mL}-1$ berberine hydrochloride solution prepared with methanol (internal standard, IS) and $400 \mu \mathrm{L}$ of acetonitrile were added successively to a $200 \mu \mathrm{L}$ plasma sample, after which the samples were thoroughly vortexed for $5 \mathrm{~min}$. The mixture was then centrifuged at $13000 \mathrm{rpm}$ for $10 \mathrm{~min}$ and the supernatant layer was transferred to a clean 1.5-mL PE tube for drying under a gentle nitrogen steam. The residue was reconstituted in $200 \mu \mathrm{L}$ methanol with the help of a vortex mixer, and after centrifugation, $25 \mu \mathrm{L}$ of the supernatant was injected for HPLC analysis. The HPLC system consisted of a Shimadzu LC-10AT pump, a SPD-10A ultraviolet-visible detector and a C18 column $(250 \times 4.6 \mathrm{~mm}$ i.d., $5.0 \mu \mathrm{m}$, Japan). The mobile phase was a mixture of acetonitrile and $10 \mathrm{mmol} \mathrm{L}^{-1}$ ammonium acetate at a volume ratio of 50/50 (V/V). The flow rate was 1.0 
$\mathrm{mL} \mathrm{min}^{-1}$ and the detection wavelength was set at $280 \mathrm{~nm}$. Quantification was performed according to the peak area ratio of the drug to internal standard ( $\left.Y=A_{\text {drug }} / A_{\mathrm{IS}}\right)$. The obtained calibration equation $(Y=0.0575 c-0.0046, n=6)$ showed good linearity over the concentration range of $0.5-30.0 \mu \mathrm{g} \mathrm{mL} \mathrm{mL}^{-1}$ with the correlation coefficient of over 0.99 . The lowest limit of quantification (LLOQ) for risperidone determination in rat plasma was $0.25 \mu \mathrm{g}$ $\mathrm{mL}^{-1}$. Accuracy of risperidone determination in rat plasma was found to be $106.26 \pm 7.75 \%$ $(n=9)$. Within-day and between-day precisions were both lower than $10.0 \%$. Mean extraction recovery of risperidone in rat plasma was over $90.0 \%$.

\section{Pharmacokinetic analysis}

Pharmacokinetic analysis was performed by a model-independent method using the 3P97 pharmacokinetic program, which was issued by the Chinese State Food and Drug Administration for pharmacokinetic analysis. The elimination rate constant $\left(K_{\mathrm{el}}\right)$ was calculated by log-linear regression of risperidone concentration and time data during the elimination phase, and the terminal half-life $\left(t_{1 / 2}\right)$ was calculated as $0.693 / K_{\mathrm{el}}$. Peak concentration $\left(c_{\max }\right)$ of the drug in plasma as well as the time to reach $c_{\max }\left(t_{\max }\right)$ were observed as raw data. The area under the plasma concentration-time curve $\left(A U C_{0-t}\right)$ from time zero to the time of the last measured concentration $\left(c_{t}\right)$ was calculated by means of the linear trapezoidal rule. $A U C_{0-\infty}$ was determined by adding $A U C_{0-t^{\prime}}$ and the extrapolated area was determined by calculating $c_{\mathrm{t}} / \mathrm{K}_{\mathrm{el}}$. The analysis and comparison of the pharmacokinetic parameters of nasal and oral administrations of risperidone were performed using the SPSS statistical software (version 22.0, SPSS Inc.). $p<0.05$ was taken as statistically significant.

\section{Nasal ciliotoxicity studies}

The $0.5 \%$ risperidone nasal gel was freshly prepared before the experiment. To investigate the effect of $\mathrm{DM} \beta \mathrm{CD}$ as well as the preservative alone in the formulation on nasal ciliary movement, $15 \% \mathrm{DM} \beta \mathrm{CD}$ solution and $0.5 \%$ chlorobutanol solution were also prepared as test solutions. Further, $0.9 \%(\mathrm{~m} / \mathrm{V})$ normal saline (NS) and $1 \%(\mathrm{~m} / \mathrm{V})$ sodium deoxycholate (a known nasal ciliotoxicity agent) were used as negative and positive controls, respectively. Nasal ciliotoxicity studies were carried out using the toad palate model method as described by Jiang et al. (10). In brief, the upper palate mucosa of toads (30-50 g, Experimental Animal Center of Inner Mongolia Medical University, China) was dissected into small patches of the same size $(3 \times 3 \mathrm{~mm})$, and then rinsed with saline. Mucosa samples were spread on a glass slide and instantly treated with a suitable amount of risperidone nasal gel or $0.2 \mathrm{~mL}$ of other test solutions. After that, they were examined under an optical microscope (Olympus, Japan) and the time of ciliary movement (LTCM) was recorded. Once the ciliary movement of the mucosa sample ceased, the sample was immediately rinsed with NS and its LTCM continued to be observed. The total LTCM for the sample will be the sum of the two recorded LTCMs.

\section{RESULTS AND DISCUSSION}

Risperidone was successfully solubilized by complexation with $\mathrm{DM} \beta \mathrm{CD}$ and formulated as a nasal gel preparation. The optimized formulation consisted mainly of the drug, Carbopol 940, propylene glycol, DM $\beta C D$, chlorobutanol and triethanolamine. The pharma- 
cokinetics and relative bioavailability of the risperidone nasal formulation were investigated in rats. Nasal administration studies demonstrated that the drug was very rapidly absorbed through the nasal mucosa of rats and its bioavailability was up to about $1600.0 \%$ relative to the oral route. The in vitro effect of the drug nasal gel as well as its main excipients on the ciliary movement of the toad palate was also explored. The ciliotoxicity of DM $\beta C D$ as well as that of chlorobutanol appeared to be moderate. The whole drug nasal formulation exhibited mild cilitoxicity and the adverse effect was temporary and reversible.

\section{Formulation of risperidone nasal gel}

Because of the limited volume of human nasal cavity, lack of adequate aqueous solubility is often the main problem in the development of nasal formulations for poorly watersoluble drugs. Based on clinical therapeutical doses of marketed oral preparations of risperidone, the nasal gel drug concentration was established to be $0.5 \%(\mathrm{~m} / \mathrm{V})$, which is equivalent to $1 \mathrm{mg}$ of drug per $0.2 \mathrm{~g}$ of nasal gel preparation. Since risperidone was reported to include different cyclodextrin derivatives in aqueous solution (11), as solubilizing agents, $\mathrm{DM} \beta \mathrm{CD}$ was utilized in nasal formulations to prepare a solution-type nasal gel of the drug. In addition, this material may also produce an absorption enhancing effect in nasal absorption of risperidone, since this effect appears to be most pronounced for $\mathrm{DM} \beta \mathrm{CD}$, as reported by Yang et al. (12). Results of the formulation sieving experiment performed by our research group have confirmed that a stable solution-type nasal gel of risperidone could be obtained only when the $\mathrm{DM} \beta \mathrm{CD}$ concentration in the nasal formulation was higher than or equal to $15 \%$.

Further, according to the bacteriostatic concentration of chlorobutanol often used in injections and ophthalmic preparations, concentrations of $0.5 \%$ of the preservative were selected for the risperidone nasal preparation. Our previous research proved that risperidone showed high oil-water partition coefficient (lipophilicity) and good chemical stability when the medium $\mathrm{pH}$ was about 6.0, and under the same $\mathrm{pH}$ conditions, chlorobutanol possessed good chemical stability as well as a bacteriostatic effect. Furthermore, Carbopol 940 exhibits high viscosity above $\mathrm{pH}$ 6.0, and to prevent and reduce irritation to nasal mucosa, the ideal $\mathrm{pH}$ of a nasal formulation should be within 4.5-6.5. Thus, it seems reasonable that the $\mathrm{pH}$ of risperidone nasal gel was finally adjusted to about 6.0. Lastly, the obtained nasal gel of risperidone with $0.35 \%$ Carbopol 940 exhibited good appearance, spreadability, and rapid in vitro release behavior.

\section{Pharmacokinetic studies}

The mean plasma concentration-time profiles of risperidone following nasal and oral administrations are illustrated in Fig. 1 while the main pharmacokinetic parameters of the drug are summarized in Table I. Very rapid absorption of risperidone after nasal administration was observed in rats and $t_{\max }$ was only approximately $5 \mathrm{~min}$. In contrast, the maximal plasma concentration $\left(c_{\max }\right)$ was reached in about 30 min when the drug was given to rats orally. The above results indicate that risperidone was more easily absorbed in rats through the nasal route than by oral administration. Furthermore, the obtained $c_{\max }$ for the nasal route at a dose of $3.0 \mathrm{mg} \mathrm{kg}^{-1}$ was found to be approximately 4 times higher than that for oral dosing at a dose of $30 \mathrm{mg} \mathrm{kg}^{-1}$. The relative bioavailability of risperidone nasal gel to oral dosing was calculated to be up to $1600.0 \%$, suggesting that nasal dosing resulted in about 15 -fold higher extent of drug absorption than the oral route. The reasons 


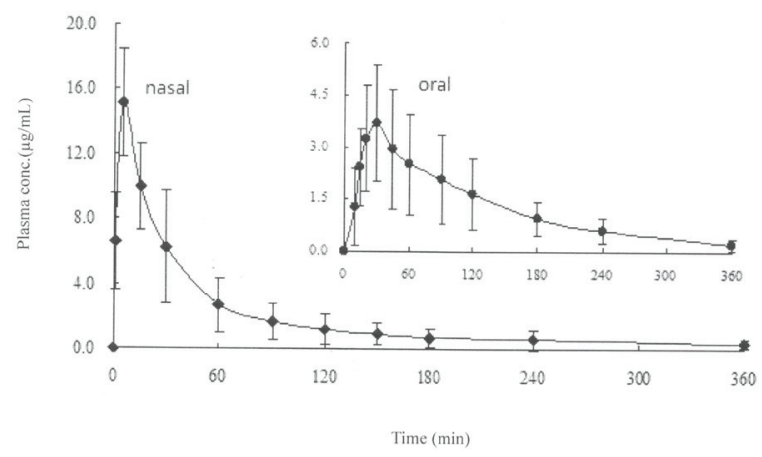

Fig. 1. Mean plasma concentration-time profiles after administration of $3.0 \mathrm{mg} \mathrm{kg}^{-1}$ of risperidone nasal gel and $30 \mathrm{mg} \mathrm{kg}^{-1}$ of the oral drug suspension to rats. Data are represented as mean $\pm \mathrm{SD}(n=6)$.

Table I. Pharmacokinetic parameters of risperidone nasal gel in rats using oral suspension as control

\begin{tabular}{|c|c|c|}
\hline Parameters & Oral suspension & Nasal gel \\
\hline$t_{\max }(\min )$ & 30.0 & 5.0 \\
\hline$c_{\max }\left(\mathrm{g} \mathrm{mL}^{-1}\right)$ & $3.6 \pm 1.4$ & $15.2 \pm 3.3^{\mathrm{a}}$ \\
\hline$A U C_{0-t}\left(\mu \mathrm{g} \mathrm{h} \mathrm{mL}^{-1}\right)$ & $7.6 \pm 4.2$ & $11.5 \pm 5.9^{\mathrm{a}}$ \\
\hline$A U C_{0-\infty}\left(\mu \mathrm{g} \mathrm{h} \mathrm{mL^{-1 } )}\right.$ & $7.9 \pm 4.5$ & $12.8 \pm 6.6^{\mathrm{a}}$ \\
\hline$F_{\mathrm{r}}(\%)$ & \multicolumn{2}{|c|}{$1608.3 \%$} \\
\hline
\end{tabular}

Data are represented as mean $\pm \mathrm{SD}(n=6)$. Oral dose $30 \mathrm{mg} \mathrm{kg}^{-1}$; nasal dose $3.0 \mathrm{mg} \mathrm{kg}^{-1}$.

${ }^{a} p<0.01$, compared to the control.

$A U C_{0-t}$ - area under the plasma concentration-time curve from time zero to the time of last measured concentration, $A U C_{0-\infty}-$ area under the plasma concentration-time curve from time zero to infinite, $c_{\max }$ - peak concentration; $t_{\max }$ - time to reach peak concentration.

for the fast absorption and good bioavailability of risperidone administrated nasally are probably as follows. Firstly, higher bioavailability of the nasally administered drug might be attributed to the avoidance of hepatic first pass metabolism compared to oral dosing of risperidone. Secondly, increased permeability of the nasal mucosa by $\mathrm{DM} \beta \mathrm{CD}$ could also improve the absorption of the drug, since numerous studies have demonstrated the efficient nasal permeation enhancing effect of cyclodextrin for different drugs (13). Lastly, since Carbopol 940 could increase the viscosity of the formulation and form a hydrogen bond with the nasal mucosa, this also resulted in increased nasal residence time of the formulation and thus improved bioavailability (14).

\section{Nasal cilitoxicity studies}

As shown in Table II, the LTCM of the toad palate treated with $0.5 \%$ risperidone nasal gel was found to be $50 \mathrm{~min}$, which was far shorter than that of the palate sample treated with the negative control $0.9 \%$ saline. However, while the toad palate was rinsed with 0.9 
Table II. Effect of risperidone nasal gel and its excipients on the toad palate cilia movement

\begin{tabular}{lcccc}
\hline Sample & LTCM (min) & $\begin{array}{c}\text { LTCM after } \\
\text { rinsing (min) }\end{array}$ & $\begin{array}{c}\text { Total LTCM } \\
(\mathrm{min})\end{array}$ & $\begin{array}{c}\text { Relative } \\
\text { percentage (\%) }\end{array}$ \\
\hline $0.9 \%$ NS & $287.8 \pm 19.4$ & - & $287.8 \pm 19.4$ & 100 \\
$1 \%$ Sodium deoxycholate & $6.6 \pm 0.8$ & 0 & $6.6 \pm 0.8^{\mathrm{b}}$ & 2.3 \\
$0.5 \%$ Risperidone nasal gel & $51.0 \pm 5.4$ & $224.2 \pm 14.6$ & $275.2 \pm 16.8^{\mathrm{a}}$ & 95.6 \\
$15 \%$ DM $\beta$ CD solution & $38.5 \pm 3.3$ & $196.0 \pm 14.8$ & $234.5 \pm 15.5^{\mathrm{b}}$ & 81.5 \\
$0.5 \%$ Chlorobutanol solution & $35.3 \pm 1.6$ & $125.6 \pm 5.1$ & $165.2 \pm 4.8^{\mathrm{b}}$ & 55.6 \\
\hline
\end{tabular}

Data are represented as mean \pm SD $(n=6)$.

${ }^{\mathrm{a}} p>0.005,{ }^{\mathrm{b}} p<0.05$ or 0.01 compared to the negative control.

$\%$ saline immediately after its mucociliary movement ceased, the mucociliary movement of the sample was soon restored and lasted about $240 \mathrm{~min}$. Total LTCM for the sample was approximately $280 \mathrm{~min}$, which was fairly close to the duration for the $0.9 \%$ saline sample. The mucocilliary movement of the toad palate treated with $1 \%$ sodium deoxyycholate solution stopped almost promptly. In addition, among the additives utilized in the risperidone nasal formulation, the preservative $0.5 \%$ chlorobutanol solution showed moderate ciliotoxicity, but the adverse effect was reversible. Further, the effects of $15 \% \mathrm{DM} \beta \mathrm{CD}$ solution on the mucociliary movement of the toad palate were found to be similar to the risperidone nasal gel. The results were consistent with the report referring to $\mathrm{DM} \beta C D(13)$. In other words, $\mathrm{DM} \beta \mathrm{CD}$ showed mild ciliotoxicity to mucocilliary movement, but also this adverse effect was reversible. Lastly, although the preservative chlorobutanol produced relatively moderate ciliotoxcity, the whole nasal gel formulation of the drug showed only mild nasal ciliotoxicity and the undesirable effect was temporary and reversible. One of the main reasons might be that the complexation phenomenon of chlorobutanol with $\mathrm{DM} \beta \mathrm{CD}$ took place in the nasal gel formulation, resulting in the lower-concentration preservative contact with the nasal mucosa (15). On the other hand, since the absorption rate of the risperidone gel following nasal dosing was very rapid, for example, $t_{\max }$ was only $5 \mathrm{~min}$ in the rat pharmacokinetic study, it seems reasonable to predict that the in vivo ciliotoxicity of the nasal gel formulation should be lower than that observed in the toad palate experiment.

\section{CONCLUSIONS}

Nasal dosing of risperidone may be an ideal alternative for the oral route and the drug nasal gel preparation will also be a promising novel prescription used in the clinical treatment of schizophrenia, with many advantages such as rapid absorption, onset of action, high bioavailability, ease of use, and self-medication. A further study is necessary to investigate the pharmacokinetics and bioavailability in humans, especially the brain targeting characteristics of the risperidone gel following nasal administration. Furthermore, the effect of the drug nasal preparation on the human nasal ciliary movement and nasal epithelial function remain to be investigated as well. Finally, whether DM $\beta C D$ in the nasal prep- 
aration will significantly increase the absorption of the drug is still unknown, and a transmucosal absorption study is underway in our laboratory.

Acknowledgements. - This study was supported by Grants from the Natural Scientific Foundation of Inner Mongolia (Grant No. 2015MS (LH) 0801) and the Scientific and Technological Million Project of Inner Mongolia Medical University (Grant No. YKD2014KJBW012).

\section{REFERENCES}

1. T. Cabaleiro, D. Ochoa, R. López-Rodríguez, M. Román, J. Novalbos, C. Ayuso and F. Abad-Santos, Effect of polymorphisms on the pharmacokinetics, pharmacodynamics, and safety of risperidone in healthy volunteers, Hum. Psychopharmacol. 29 (2014) 459-469; DOI: 10.1002/hup.2420.

2. J. L. Sun, Q. X. Chen, S. Tian, F. R. Meng, Y. Lv and G. H. Du, Absorption of risperidone polymorphs administrated orally in rats, Chin. Pharm. J. 46 (2011) 1919-1922.

3. T. K. Vyas, A. Shahiwala, S. Marathe and A. Misra, Intranasal drug delivery for brain targeting, Curr. Drug Deliv. 2 (2005) 165-175; DOI: 10.2174/1567201053586047.

4. C. R. Behl, H. K. Pimplaskar, A. P. Sileno, J. deMeireles and V. D. Romeo, Effects of physicochemical properties and other factors on systemic nasal drug delivery, Adv. Drug Deliv. Rev. 29 (1998) 89-116; DOI: doi:10.1016/S0169-409X(97)00063-X.

5. M. Kumar, A. Misra, A. K. Babbar, A. K. Mishra, P. Mishra and K. Pathak, Intranasal nanoemulsion based brain targeting drug delivery system of risperidone, Int. J. Pharm. 358 (2008) 285-291; DOI: 10.1016/j.ijpharm.2008.03.029.

6. W. N. Ma and F. G. Gu, Effect of $\mathrm{pH}$ on the chemical stability, solubility and oil-water partition coefficient of risperidone, Centr. South Pharm. 13 (2015) 473-476; DOI: 10.7539/j.issn.16722981.2015.05.006.

7. H. S. Mahajan, S. K. Shah and S. J. Surana, Nasal in situ gel containing hydroxy propyl $\beta$-cyclodextrin inclusion complex of arthemeter: development and in vitro evaluation, J. Incl. Phenom. Macrocycl. Chem. 70 (2011) 49-58; DOI: 10.1007/s10847-010-9861-x.

8. C. Tas, C. K. Ozkan, A. Savaser, Y. Ozkan, U. Tasdemir and H. Altunay, Nasal absorption of metoclopramide from different Carbopol 981 based formulations: In vitro, ex vivo and in vivo evaluation, Eur. J. Pharm. Biopharm. 64 (2006) 246-254; DOI: 10.1016/j.ejpb.2006.05.017.

9. F. G. Gu, F. D. Cui and Y. L. Gao, Preparation of prostaglandin E1-hydroxypropyl- $\beta$-cyclodextrin complex and its nasal delivery in rats, Int. J. Pharm. 290 (2005) 101-108; DOI: 10.1016/j. ijpharm.2004.11.021.

10. X. G. Jiang, J. B. Cui, X. L. Fang, Y. Wei and N. Z. Xi, Toxicity of drugs on nasal mucocilia and the method of its evaluation, Acta. Pharm. Sin. 30 (1995) 848-853; DOI: 10.3321/j.issn:05134870.1995.11.009.

11. M. Jug, I. Kos and M. Bećirević-Laćan, The pH-dependent complexation between risperidone and hydroxypropyl- $\beta$-cyclodextrin, J. Incl. Phenom. Macrocycl. Chem. 64 (2009) 163-171; DOI: 10.1007/ s10847-009-9549-2.

12. T. Yang, A. Hussain, J. Paulson, T. J. Abbruscato and F. Ahsan, Cyclodextrinsin nasal delivery of low molecular weight heparins: in vivo and in vitro studies, Pharm. Res. 21 (2004) 1127-1136; DOI: 10.1023/B:PHAM.0000032998.84488.7a.

13. E. Marttin, J. C. Vethoef and F. W. H. M. Merkus, Efficacy safety and mechanism of cyclodextrins as absorption enhancers in nasal delivery of peptide and protein drugs, J. Drug Targeting 6 (1998) 17-36; DOI: 10.3109/10611869808997878.

14. G. Rathnam, N. Narayanan and R. Ilavarasan, Carbopol-based gels for nasal delivery of progesterone, AAPS Pharm. Sci. Tech. 9 (2008) 1078-1082; DOI: 10.1208/s12249-008-9144-7.

15. F. G.. Gu, D. Hao, G. D. L. Meng, C. Z. Wu and Y. Wang, Preparation of bisoprolol fumarate nasal spray and its nasal delivery in rats, Pak. J. Pharm. Sci. 28 (2015) 2173-2178. 